\title{
Management of hepatocellular carcinoma: time to be resilient
}

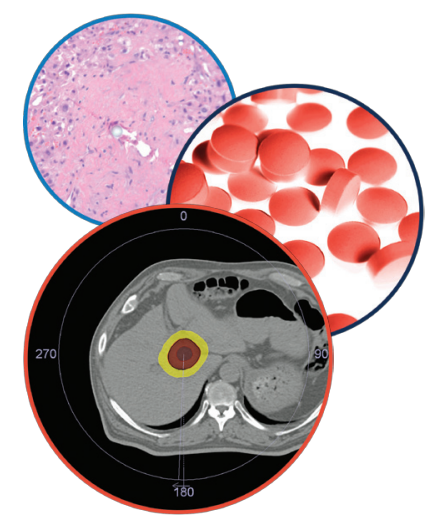

Hepatic Oncology

Jean-Francois Dufour*

\section{European Association for the Study of the Liver Hepatocellular Carcinoma Summit}

\section{3-14 February 2014, Geneva, Switzerland}

Following on from the publication of clinical practice guidelines [1], the European Association for the Study of the Liver (EASL) organized a 2-day conference focused on the management of hepatocellular carcinoma (HCC). Over 700 delegates convened in Geneva in mid-February 2014. This report selects a few important points that were discussed at the meeting.

In comparison to other tumors, where the use of biomarkers has become routine in guiding clinical practice, none of them are used in HCC and the field is lagging behind. Several groups have published molecular classifications of HCCs, which are informative, and help us better understand the biology of HCC. Fortunately, different groups, using different samples, have identified the same HCC classes. The two common classes are characterized by:

- Mutations of $\beta$-catenin;

- Phosphorylation of the IGF receptor, phosphorylation of AKT, and mTOR activation.

The latter can be further divided into two subclasses, one coming from progenitor cells (high alpha-fetoprotein [AFP] at early stage), and the other, characterized by the activation of Wnt/TGF- $\beta$ [2]. In terms of prognosis, the recurrence of HCC can be predicted by a five-gene tumor signature and a 186-gene genetic signature derived from adjacent nontumor tissue [3].

Liver transplantation is an established treatment option for patients within the Milan criteria, and patients have shown 5 -year survival rates above $60 \%$. Those criteria have a high level of evidence and a strong grade of recommendation, in contrast to extended criteria and downstaging. The decision-making with respect to liver transplantation for liver cancer versus nonliver cancer is a relatively recent issue due to the continuing increase in the proportion of patients listed with HCC. These two categories of patients, those listed for decompensated cirrhosis and those listed for HCC follow two conflicting principles: the urgency principle and the utility principle. Patients listed for decompensated cirrhosis are under the urgency principle: they have a high pretransplantation mortality and a high chance of recovery after transplantation; they do not have competitive options; and they can be listed according to a predictable outcome calculated with the Model of End-stage Liver Disease (MELD) score. Patients listed for HCC have low mortality on the waiting list, bear a risk of recurrence after transplantation, have competitive therapeutic options and lack a good prognostic score. There are subgroups of patients within the Milan criteria with different risk of dropout from the list [4]. Steady increase in AFP identifies patients at high risk of dropout [5]. For HCC less than $2 \mathrm{~cm}$ there is the 'ablate and wait' strategy, which carries two important benefits: first, it reduces the risk of dropout; and second, it spares organs for those who do not have recurrence.

*Department of Clinical Pharmacology, University of Berne, Berne, Switzerland; jean-francois.dufour@ikp.unibe.ch
Future $\because$ Medicine ${ }_{\text {part of }}$ 
For Child A patients with a small tumor, no transplant options prevail, which allows the list to be driven by the concept of urgency. As the tumor burden increases and the liver cirrhosis progresses, the option to transplant prevails because it provides a survival benefit to a point where the utility concept kicks in and oblige to disregard transplantation [6].

The clinical trial landscape in HCC has been a source of frustration in recent years. Since the publication in 2008 of the two positive randomized clinical trials that led to the registration of sorafenib, numerous trials have been conducted and all of them have been negative. Promising drugs, such as erlotininb, sunitinib, linifanib, brivanib and everolimus, have failed to show any advantage in HCC. As with other tumor types, it is evident that there is a need to identify predictors of response, so that trials may be populated with patients who are likely to demonstrate an effect. In the literature, about 140 mutation-driver genes, oncogenes and tumor suppressors have been described; about 11 of these genes have been consistently associated with HCC. Activating NRAS and KRAS mutations, which result in an overactivation of the ERK pathway, occur in about $5 \%$ of HCCs. Refametinib is an oral inhibitor of mitogen-activated protein kinase 1 and 2, which belong to the ERK pathway. A proofof-concept trial that enrols only patients with $N R A S$ and KRAS mutations can be designed to assess refametinib. The second-line Phase II trial testing tivantinib was globally negative, but a post-hoc analysis revealed a striking survival advantage for patients with a high tumor expression of c-met [7]. Patients treated with tivantinib with low expression of c-met had a survival of 3.8 months, whereas those with high expression of c-met had a survival of 7 months, which is almost double. Based on this finding, a Phase III, randomized, double-blind study has been initiated. Retrospective analyses looking for predictive biomarkers have also been performed using data from the sorafenib registration trial. Basal c-kit and HGF level were independent predictors of survival in the sorafenib arm, but the level of significance required for these to be considered as predictive biomarkers for sorafenib response was not achieved [8].

The majority of patients diagnosed with HCC are in the intermediate stage, Barcelona Clinic Liver Center, and guidelines recommend transarterial chemoembolization (TACE) for these patients. TACE has been shown to confer a survival advantage. The mechanism of action of TACE, which is to produce anoxia and necrosis, results in the creation of a border of hypoxic tissue. Hypoxia is a potent angiogenic stimulus. The rational to combine TACE with systemic antiangiogenic therapy has been outlined in a previous EASL monothematic session on HCC [9]. Since then, several trials have been conducted. The largest randomized trial that tested TACE with drug-eluting beads, with or without sorafenib, unfortunately, reported no clinically significant advantage. This setback raised several important conceptual questions. Is the benefit of TACE so large that it hides the smaller benefit of the antiangiogenic therapy? Are the patients who are selected for TACE not benefiting from sorafenib? How can one approach negatively affect the second approach? In order to exploit meaningfully this combination these questions would have to be answered.

Best supportive care is a neglected area of HCC care and research. However, this is one of the most important treatments as most patients will, at some point, migrate towards this treatment category. The primary goal of best supportive care is to improve and maintain patient quality of life. This requires physicians to show a willingness to address symptoms, such as sleep disturbances, anxiety and fatigue. Simple behavioral interventions may substantially alleviate these symptoms. Patients need to be encouraged to have structured activities during the day, which includes physical activity. These nonpharmacologic approaches may substantially improve sleep quality and decrease the feeling of fatigue. Moreover, physical activity has been shown to reduce the rate of tumor recurrence in patients with breast carcinoma and colorectal tumor. Frequently, patients with advanced HCC drastically change their alimentary habits by becoming strict teetotallers, even if alcohol has never been a problem in their life, and renouncing food they like. Malnutrition is frequent and patients should not worsen their quality of life by depriving themselves from reasonable culinary pleasures. In a randomized controlled trial, megestrol acetate did not improve survival, nor quality of life, but did improve emotional functioning, nausea/vomiting, and appetite loss. Pain can be an issue in case of bone metastases. Cementoplasty should be discussed in case of vertebral pain, and irradiation is another option that may be considered. These options should be 
implemented in parallel with an adequate analgesia. As first-line treatment, paracetamol should be prescribed rather than nonsteroidal antiinflammatory drugs. If the pain persists, opioids should be initiated, such as hydromorphone and fentanyl.

This is a subjective selection of highlights from this meeting. Thanks to the EASL webcasting effort, all the presentations can be accessed via the internet for those interested to learn more from this meeting.
Financial \& competing interests disclosure

The author wishes to thank advisory committees: Bayer, BMS, Gilead Science, Janssen Cilag, Jennerex, Merck, Novartis and Roche, as well as those speaking and teaching: Bayer, Boehringer-Ingelheim, Novartis and Roche. The author has no other relevant affliations or financial involvement with any organization or entity with a financial interest in or financial conflict with the subject matter or materials discussed in the manuscript apart from those disclosed.

No writing assistance was utilized in the production of this manuscript.

\section{References}

1 EASL-EORTC clinical practice guidelines: management of hepatocellular carcinoma. J. Hepatol. 56, 908-943 (2012).

2 Hoshida Y, Nijman SM, Kobayashi M et al. Integrative transcriptome analysis reveals common molecular subclasses of human hepatocellular carcinoma. Cancer Res. 69, 7385-7392 (2009).

3 Nault JC, De Reynies A, Villanueva A et al. A hepatocellular carcinoma 5-gene score associated with survival of patients after liver resection. Gastroenterology 145, 176-187 (2013).
4 Mehta N, Dodge JL, Goel A et al. Identification of liver transplant candidates with hepatocellular carcinoma and a very low dropout risk: implications for the current organ allocation policy. Liver Transpl. 19, 1343-1353 (2013).

5 Vibert E, Azoulay D, Hoti E et al. Progression of alphafetoprotein before liver transplantation for hepatocellular carcinoma in cirrhotic patients: a critical factor. Am. J. Transplant. 10, 129-137 (2010).

6 Bruix J, Gores GJ, Mazzaferro V. Hepatocellular carcinoma: clinical frontiers and perspectives. Gut 63, 844-855 (2014).
7 Santoro A, Rimassa L, Borbath I et al. Tivantinib for second-line treatment of advanced hepatocellular carcinoma: a randomised, placebo-controlled Phase 2 study. Lancet Oncol. 14, 55-63 (2013).

8 Llovet JM, Pena CE, Lathia CD et al. Plasma biomarkers as predictors of outcome in patients with advanced hepatocellular carcinoma. Clin. Cancer Res. 18, 2290-2300 (2012).

9 Dufour JF, Johnson P. Liver cancer: from molecular pathogenesis to new therapies: summary of the EASL single topic conference. J. Hepatol. 52, 296-304 (2010). 